\title{
BAUTIN IDEALS AND TAYLOR DOMINATION
}

\author{
Y. YOMDIN
}

\begin{abstract}
We consider families of analytic functions with Taylor coefficients-polynomials in the parameter $\lambda: f_{\lambda}(z)=\sum_{k=0}^{\infty} a_{k}(\lambda) z^{k}, a_{k} \in \mathbb{C}[\lambda]$. Let $R(\lambda)$ be the radius of convergence of $f_{\lambda}$. The "Taylor domination" property for this family is the inequality of the following form: for certain fixed $N$ and $C$ and for each $k \geq N+1$ and $\lambda$,

$$
\left|a_{k}(\lambda)\right| R^{k}(\lambda) \leq C \max _{i=0, \ldots, N}\left|a_{i}(\lambda)\right| R^{i}(\lambda) .
$$

Taylor domination property implies a uniform in $\lambda$ bound on the number of zeroes of $f_{\lambda}$. In this paper we discuss some known and new results providing Taylor domination (usually, in a smaller disk) via the Bautin approach. In particular, we give new conditions on $f_{\lambda}$ which imply Taylor domination in the full disk of convergence. We discuss Taylor domination property also for the generating functions of the Poincaré type linear recurrence relations.
\end{abstract}

2010 Mathematics Subject Classification: 34C05, 34C25, 30B10.

Key words: Bautin ideals, Taylor domination, Turán Lemma, Poincaré-type recurrence.

\section{Introduction}

Consider a family of analytic functions $f_{\lambda}(z), \lambda \in \mathbb{C}^{m}$, represented by a convergent power series with the coefficients-polynomials in $\lambda$ :

$$
f_{\lambda}(z)=\sum_{k=0}^{\infty} a_{k}(\lambda) z^{k}, \quad a_{k} \in \mathbb{C}[\lambda] .
$$

The main problem we discuss in this paper is the following: under what conditions $f_{\lambda}$ possesses the "Taylor domination" property, i.e. the inequality for the Taylor coefficients $a_{k}(\lambda)$ of the following form (see Section 3 for an accurate definition): for certain fixed $N$ and $C$ and for each $k>N$ and $\lambda$,

$$
\left|a_{k}(\lambda)\right| R^{k}(\lambda) \leq C \max _{i=0, \ldots, N}\left|a_{i}(\lambda)\right| R^{i}(\lambda) .
$$

This research was supported by the ISF, grant no. 639/09 and by the Minerva foundation. 
Taylor domination property is well known to be essentially equivalent to the bound on the number of solutions of $f_{\lambda}(z)=c($ see $[\mathbf{4}, \mathbf{1 7}, \mathbf{2 2}])$. In this paper we discuss some known $[\mathbf{7}, \mathbf{9}, \mathbf{1 0}, \mathbf{2 5}]$ and new results which derive Taylor domination from algebraic properties of the Bautin ideal $I\left(f_{\lambda}\right)=\left\{a_{0}(\lambda), \ldots, a_{k}(\lambda), \ldots\right\}$. In particular, we give conditions on $f_{\lambda}$ which imply Taylor domination in the full disk of convergence. We discuss Taylor domination property also for the generating functions of the Poincaré type linear recurrence relations, presenting a result recently obtained in $[\mathbf{1}]$.

Our main motivation is in study of closed trajectories of differential equations. Here the pioneering approach suggested by Bautin in $[\mathbf{2}, \mathbf{3}]$ has already provided some of the strongest results available. We believe that further development of this approach may improve our understanding of the structure of closed trajectories, and hope that the present paper, as well as [1], can be considered as certain steps in this direction.

\section{Motivation: Smale-Pugh problem}

We consider Abel differential equation

$$
y^{\prime}=p(x) y^{2}+q(x) y^{3}
$$

with polynomial coefficients $p, q$ on the interval $[a, b]$. A solution $y(x)$ of $(2.1)$ is called "closed" if $y(a)=y(b)$. The Smale-Pugh problem is to bound the number of isolated closed solutions of (2.1) in terms of the degrees of $p$ and $q$. This problem is a version of the (second part of) Hilbert's 16-th problem asking for the bound of the number of isolated closed trajectories of a polynomial system in the plane. The SmalePugh problem has appeared in the course of the work of S. Smale's seminar in the beginning of 1960 's, in an attempt to find "the simplest situation where Hilbert's 16-th problem is still non-trivial". There are many reasons today to consider this choice as highly successful - see $[\mathbf{6}]-[\mathbf{8}],[\mathbf{1 1}]-[\mathbf{1 4}],[\mathbf{1 8}]$ and references therein as a very partial evidence.

The problem above can be naturally expressed in terms of the Poincaré "first return" map $y_{b}=G\left(y_{a}\right)$ along $[a, b]$. Let $y\left(x, y_{a}\right)$ denote the solution $y(x)$ of $(2.1)$ satisfying $y(a)=y_{a}$. The Poincaré map $G$ associates to each initial value $y_{a}$ at $a$ the value $y_{b}$ at $b$ of the solution $y\left(x, y_{a}\right)$ analytically continued along $[a, b]$. Closed solutions correspond to the fixed points of $G$. So the problem is reduced to bounding the number of the fixed points of $G$, or of zeroes of $G(y)-y$. 
However, this last problem turns out to be extremely difficult. The Poincaré map, which "predicts the future", is one of the most mysterious functions in Analysis. It does not render itself to any convenient analytic representation, and hence most of the known approaches to "bounding zeroes" are not applicable. Historically, one of the most successful directions in the study of the Poincaré map $G$ was the direction initiated by Bautin in $[\mathbf{2}, \mathbf{3}]$ : to derive the analytic properties of $G$, in particular, the number of its fixed points, from the structure of its Taylor coefficients.

It is well known that $G(y)$ for small $y$ is given by a convergent power series

$$
G(y)=y+\sum_{k=2}^{\infty} v_{k}(p, q, a, b) y^{k} .
$$

The "Poincaré coefficients" $v_{k}(p, q, x, a)$ of the Poincaré map from $x$ to $a$ satisfy the following differential-recurrence relation (see, for example, $[6])$ :

(2.3) $\frac{d v_{k}}{d x}=-(k-1) p v_{k-1}-(k-2) q v_{k-2}, \quad v_{0} \equiv 0, v_{1} \equiv 1, v_{k}(0)=0, k \geq 2$.

It is easy to see from $(2.3)$ that the Poincaré coefficients $v_{k}(p, q, a, b)$ are polynomials with rational coefficients in the parameters of the problem (i.e. in the coefficients of $p$ and $q$ ). They can be explicitly computed for as large indices $k$ as necessary.

It is more difficult to find general patterns in the structure of $v_{k}$. It is known that each $v_{k}(p, q, a, b)$ can be expressed as linear combinations of certain iterated integrals of $p$ and $q$ along $[a, b]$ (see, for example, $[6]$ ). In some aspects the generalized moments $m_{k}=\int_{a}^{b} P^{k}(x) q(x) d x$ provide a rather accurate approximation of $v_{k}$ (see [6]). But in general a much better understanding of the structure of $v_{k}$ is required in order to provide even "semi-local" bounds on the fixed points of the Poincaré map.

\section{Taylor domination}

Let $f(z)=\sum_{k=0}^{\infty} a_{k} z^{k}, a_{k} \in \mathbb{C}$, be a series with the radius of convergence $\widehat{R}>0$.

Definition 3.1. Let a positive $R \leq \widehat{R}$, a natural $N$, and a positive sequence $S(k)$, growing sub-exponentially as $k$ tends to infinity, be fixed. The function $f$ possesses an $(N, R, S(k))$-Taylor domination property if for each $k \geq N+1$ we have

$$
\left|a_{k}\right| R^{k} \leq S(k) \max _{i=0, \ldots, N}\left|a_{i}\right| R^{i} .
$$


For $S(k) \equiv C$ a constant, we shall call this property $(N, R, C)$-Taylor domination.

Taylor domination allows us to compare the behavior of $f(z)$ with the behavior of the polynomial $P_{N}(z)=\sum_{k=0}^{N} a_{k} z^{k}$. In particular, the number of zeroes of $f$ can be easily bounded in this way.

Theorem 3.1 ([22, Lemma 2.2]). There exists a finite function $M\left(N, \frac{R^{\prime}}{R}, C\right)$, tending to infinity as $\frac{R^{\prime}}{R}$ tends to 1 , and equal to $N$ for $\frac{R^{\prime}}{R}$ sufficiently small, such that the following bound holds: if $f$ possesses a $(N, R, C)$-Taylor domination property then for any $R^{\prime}<R, f$ has at most $M\left(N, \frac{R^{\prime}}{R}, C\right)$ zeroes in $D_{R^{\prime}}$.

In particular, put $R_{1}=\frac{R}{4}, R_{2}=\frac{R}{2 \max (C, 2)}$ and $R_{3}=\frac{R}{2^{3 N} \max (C, 2)}$. Then the number of zeroes of $f$ in the disks $D_{R_{1}}, D_{R_{2}}$ and $D_{R_{3}}$ does not exceed $5 N+\log _{5 / 4}(2+C), 5 N+10$ and $N$, respectively.

An explicit expression for $M$ is given in [22, Proposition 2.2.2]. The corresponding bounds are true also in a general case of $(N, R, S(k))$-Taylor domination. Indeed, it is easy to see that an $(N, R, S(k))$-domination implies for each $R^{\prime}<R$ an $\left(N, R^{\prime}, C\right)$-domination, with a certain constant $C$ depending on $\frac{R^{\prime}}{R}$ and on the sequence $S(k)$. See [1] for specific estimates.

It is important to stress that Taylor domination property is essentially equivalent to the bound on the number of zeroes of $f-c$, for each $c$. Let us give the following definition (see [17] and references therein):

Definition 3.2. A function $f$ regular in a domain $\Omega \subset \mathbb{C}$ is called $p$-valent there, if for any $c \in \mathbb{C}$ the number of solutions in $\Omega$ of the equation $f(z)=c$ does not exceed $p$.

Theorem 3.2 (Biernacki, [4]). If $f$ is p-valent in the disk $D_{R}$ of radius $R$ centered at $0 \in \mathbb{C}$ then

$$
\left|a_{k}\right| R^{k} \leq(A k / p)^{2 p} \max _{i=0, \ldots, p}\left|a_{i}\right| R^{i} .
$$

Here $A$ is an absolute constant.

For a detailed discussion of this and of some more accurate bounds, as well of some open problems in this line, see $[\mathbf{1 5}, \mathbf{1 6}]$.

In our notations, Theorem 3.2 claims that a function $f$ which is $p$-valent in $D_{R}$, possesses an $\left(N, R,(A k / p)^{2 p}\right)$-Taylor domination property. Theorem 3.1 shows that the inverse is also essentially true.

For univalent functions, i.e. for $p=1$, and for $a_{0}=0, R=1$, the Bieberbach conjecture proved by de Branges in [5] claims that $\left|a_{k}\right| \leq$ $k\left|a_{1}\right|$ for each $k$. 
Now we return to a family of analytic functions $f_{\lambda}(z)=\sum_{k=0}^{\infty} a_{k}(\lambda) z^{k}$, $\lambda \in \mathbb{C}^{m}$, as in (1.1), with $a_{k} \in \mathbb{C}[\lambda]$. The position of singularities, and hence the radius of convergence $R(\lambda)$, in general, depend on $\lambda$. Let a positive function $R_{1}(\lambda) \leq R(\lambda)$, a natural $N$, and a positive sequence $S(k)$, growing sub-exponentially as $k$ tends to infinity, be fixed.

Definition 3.3. The family $f_{\lambda}(z)$ possesses an $\left(N, R_{1}(\lambda), S(k)\right)$-Taylor domination property if for each $k \geq N+1$ and for each $\lambda \in \mathbb{C}^{m}$,

$$
\left|a_{k}(\lambda)\right| R_{1}^{k}(\lambda) \leq S(k) \max _{i=0, \ldots, N}\left|a_{i}(\lambda)\right| R_{1}^{i}(\lambda)
$$

with $N$ and a sub-exponentially growing sequence $S(k)$ not depending on $\lambda$. If $R_{1}(\lambda) \equiv R(\lambda)$, then $f_{\lambda}(z)$ is said to possess an $(N, R(\lambda), S(k))$-uniform Taylor domination.

Uniform Taylor domination implies a uniform in $\lambda \in \mathbb{C}^{m}$ bound on the number of zeroes in each disk $D_{\alpha R(\lambda)}$, for any fixed $\alpha<1$. Our goal is to give conditions for families $f_{\lambda}(z)$ to possess uniform Taylor domination property.

\section{Taylor domination via the Bautin ideal}

From now on we shall consider families $f_{\lambda}(z)$ as in (1.1), satisfying the following conditions:

(1) $\operatorname{deg} a_{k}(\lambda) \leq C_{1} k+C_{2}, k=0,1, \ldots$.

(2) $\left\|a_{k}(\lambda)\right\| \leq C_{3} \cdot C_{4}^{k}, k=0,1, \ldots$. Here the norm of a polynomial is defined as the sum of the absolute values of its coefficients.

Following $\left[\mathbf{7}, \mathbf{2 5}\right.$ ] we call families $f_{\lambda}(z)$ satisfying these conditions A-families.

Definition 4.1. For $f_{\lambda}(z)$ an A-family the Bautin ideal $I(f)$ is the ideal in $\mathbb{C}[\lambda]$ generated by all the Taylor coefficients $a_{k}(\lambda)$ :

$$
I(f)=\left\{a_{0}(\lambda), a_{1}(\lambda), \ldots, a_{k}(\lambda), \ldots\right\} .
$$

Because of the Noetherian property of $\mathbb{C}[\lambda]$, the ascending chain of ideals $I_{k}(f)=\left\{a_{0}(\lambda), a_{1}(\lambda), \ldots, a_{k}(\lambda)\right\}$ stabilizes for a certain index $N$ : $I_{N-1}(f) \subset I_{N}(f)=I_{N+1}(f)=\cdots$. Hence, in fact, $I(f)=I_{N}(f)$. This index $N=N(f)$ is called the Bautin index of $f$.

Bautin ideals were introduced by Bautin in $[2,3]$ in his study of the limit cycles of the plane polynomial systems which appear in small perturbations of the integrable cases. In particular, it was shown there that in perturbations of the linear center of the form $\dot{x}=-y+F(x, y)$, $\dot{y}=x+G(x, y)$ with $F(x, y)$ and $G(x, y)$ polynomials of degree two 
vanishing at the origin with their first derivatives, at most three limit cycles may appear in a sufficiently small neighborhood of the origin.

Although Bautin considered a real situation, his approach allows one to prove the following result (see $[\mathbf{9}, \mathbf{1 0}])$ :

Theorem 4.1. For $f_{\lambda}(z)$ an A-family let $N=N(f)$ be its Bautin index. Then for each $\lambda \in \mathbb{C}^{m}$ the function $f_{\lambda}(z)$ has at most $N$ zeroes in a sufficiently small neighborhood of the origin.

Sketch of the proof: For each $k \geq N+1$ we have $a_{k}(\lambda) \in I(f)=I_{N}(f)=$ $\left\{a_{0}(\lambda), a_{1}(\lambda), \ldots, a_{N}(\lambda)\right\}$. Hence

$$
a_{k}(\lambda)=\sum_{i=0}^{N} \varphi_{i}^{k}(\lambda) a_{i}(\lambda) .
$$

It is rather easy to see that, because of the conditions (1) and (2) in the definition of A-families, and via the Hironaka division algorithm, the degrees of $\varphi_{i}^{k}$ in (4.1) grow at most linearly in $k$ while their norms grow at most exponentially. Therefore for each $\lambda \in \mathbb{C}^{m}$ (4.1) implies a $\left(N, R^{\prime}(\lambda), C(\lambda)\right.$ )-Taylor domination for $f_{\lambda}(z)$ with certain constants $R^{\prime}(\lambda), C(\lambda)$, where $R^{\prime}(\lambda)$ is positive but may be much smaller than the true radius of convergence of $f_{\lambda}$. Now the result follows from Theorem 3.1 above.

A natural question is: can we explicitly estimate the size of the neighborhood in Theorem 4.1 via Taylor domination? In $[\mathbf{9 , ~ 1 0}]$ this question has been studied on the base of Hironaka's Division Theorem applied to (4.1). Rather accurate estimates have been obtained there: the radius of convergence $R(\lambda)$ is typically of order $\frac{C}{|\lambda|^{K_{1}}}$, while we can bound zeroes only in $D_{R^{\prime}(\lambda)}$ with $R^{\prime}$ of order $\frac{1}{|\lambda|^{K_{2}}}, K_{2}>K_{1}$. We believe that without additional assumptions on A-families $f_{\lambda}$, the uniform control of zeroes in the full disk of convergence cannot be achieved. An important open question is to specify an appropriate subclass of A-families, possessing such control, and wide enough to contain important examples. An attempt to give an answer in "algebraic terms", through certain "Bautin-type" ideals, was suggested in [25]. However, the conditions given in [25] are rather difficult to check, so only some "synthetic" examples were presented there. In the next section we provide much simpler (but rather restrictive) conditions on A-families, which, however, are satisfied in some important examples. 
4.1. Uniform Taylor domination via Bautin-type ideals. Consider A-families $f_{\lambda}(z)=\sum_{k=0}^{\infty} a_{k}(\lambda) z^{k}, \lambda \in \mathbb{C}^{m}$, satisfying the following conditions:

(Afam-1) The coefficients $a_{k}$ have the form $a_{k}(\lambda)=P^{k+1}(\lambda) R_{k}(\lambda)$ for a certain polynomial $P(\lambda)$, with $\operatorname{deg} R_{k}(\lambda)=d$, and with $\left\|R_{k}(\lambda)\right\| \leq S(k)$ for each $k=0,1, \ldots$. Here the norm of a polynomial is the sum of absolute values of its coefficients, and $S(k)$ is a certain sequence of sub-exponential growth.

(Afam-2) $\lim \sup _{k \rightarrow \infty}\left|R_{k}(\lambda)\right|^{\frac{1}{k}}=1$ for each $\lambda \in \mathbb{C}^{m}$.

(Afam-3) Consider the increasing chain of ideals $J_{k}=J_{k}(f)=\left\{R_{0}(\lambda), \ldots\right.$, $\left.R_{k}(\lambda)\right\}$, and let $N$ be its stabilization moment. Put $J(f)=$ $J_{N}(f)$. Then $M(\lambda)=\max _{j=0, \ldots, N}\left|R_{j}(\lambda)\right|$ satisfies $M(\lambda) \geq$ $K|\lambda|^{d}$, for each $\lambda$ with $|\lambda| \geq S$. Here $K>0, S>0$ are certain constants, and for $\lambda=\left(\lambda_{1}, \ldots, \lambda_{m}\right) \in \mathbb{C}^{m}$ by definition $|\lambda|=\max _{j=1, \ldots, m}\left|\lambda_{j}\right|$.

Remark. For one-dimensional $\lambda \in \mathbb{C}$, condition (Afam-3) follows from condition (Afam-1). Indeed, each of the polynomials $R_{j}(\lambda)$ has degree $d$ by (Afam-1). So its coefficient $\alpha_{j, d}$ is non-zero. Put $\alpha=\max _{j=0, \ldots, N}\left|\alpha_{j, d}\right|$, and let this maximum be achieved for $j=l$. Put also $S=2 \max _{j=0, \ldots, N} S(j)$. Now for $|\lambda| \geq S$ we have

$$
M(\lambda) \geq\left|R_{l}(\lambda)\right| \geq \alpha|\lambda|^{d}\left(1-S(k) \lambda^{-1}\right) \geq \frac{\alpha}{2}|\lambda|^{d} .
$$

So (Afam-3) is satisfied for $S$ as above, and for $K=\frac{\alpha}{2}$.

Theorem 4.2. Assume that an A-family $f_{\lambda}(z)$ satisfies conditions (Afam). Then:

(1) The radius of convergence $R(\lambda)$ is equal to $|P(\lambda)|^{-1}$ for each $\lambda \in$ $\mathbb{C}^{m}$

(2) The ideal $J(f)$ is $\{1\}$, the Bautin index of $f_{\lambda}(z)$ is at most $N$, and the Bautin ideal $I(f)$ contains $\left\{P^{N+1}(\lambda)\right\}$.

(3) The family $f$ possesses the $(N, R(\lambda), C S(k))$-uniform Taylor domination, with $S(k)$ the sequence in condition (Afam-1), and $C$ a constant.

Proof: We have

$$
R^{-1}(\lambda)=\limsup _{k \rightarrow \infty}\left|a_{k}(\lambda)\right|^{\frac{1}{k}}=|P(\lambda)| \limsup _{k \rightarrow \infty}\left|R_{k}(\lambda)\right|^{\frac{1}{k}}=|P(\lambda)|
$$

for each $\lambda \in \mathbb{C}^{m}$, by conditions (Afam). The ideal $J(f)$ has no zeroes, because of condition (Afam-2), and thus $J(f)$ is $\{1\}$. Next, assume that $k \geq$ $N+1$ is fixed. Because of stabilization of the ideals $J_{l}(f)$ we have $J_{k}(f)=$ 
$J_{N}(f)=J(f)$, and hence $R_{k} \in J(f)$, i.e. $R_{k}(\lambda)=\sum_{j=0}^{N} \psi_{j}(\lambda) R_{j}(\lambda)$ for certain polynomials $\psi_{j}$. Multiplying this equality by $P^{k+1}$ we obtain $a_{k}=P^{k+1} R_{k}=\sum_{j=0}^{N} P^{k-j} \psi_{j} P^{j+1} R_{j}=\sum_{j=0}^{N} P^{k-j} \psi_{j} a_{j} \in I_{N}$. Therefore the Bautin ideals $I_{j}$ stabilize at most from $j=N$ and hence the Bautin index is at most $N$. Starting with $1=\sum_{j=0}^{N} \phi_{j}(\lambda) R_{j}(\lambda)$ and repeating the calculations, we get $P^{N+1}(\lambda) \in I(f)$.

To prove the $(R(\lambda), N, C S(k))$-uniform Taylor domination, we notice that $\left|a_{k}(\lambda)\right| R^{k}(\lambda)=\left|P^{k+1}(\lambda)\right| \cdot\left|R_{k}(\lambda)\right| \cdot\left|P^{-k}(\lambda)\right|=|P(\lambda)| \cdot\left|R_{k}(\lambda)\right|$. So we have to show that there exists a constant $C$ such that for $k \geq N+1$ we have $\left|R_{k}(\lambda)\right| \leq C S(k) M(\lambda)$, where, as above, $M(\lambda)=\max _{j=0, \ldots, N}\left|R_{j}(\lambda)\right|$. We shall show this fact, bounding $\left|R_{k}\right|$ from above, and independently bounding $M$ from below. For $\left|R_{k}\right|$ we consider two cases: $|\lambda| \leq 1$ and $|\lambda|>1$. In the first case $\left|R_{k}(\lambda)\right| \leq\left\|R_{k}\right\| \leq S(k)$, and in the second case $\left|R_{k}(\lambda)\right| \leq|| R_{k}|| \cdot|\lambda|^{d} \leq S(k)|\lambda|^{d}$, by condition (Afam-1). So for any $\lambda$ we get

$$
\left|R_{k}(\lambda)\right| \leq S(k)\left(1+|\lambda|^{d}\right) .
$$

As for $M(\lambda)$, by condition (Afam-3) we have $M(\lambda) \geq K|\lambda|^{d}$, for each $\lambda$ with $|\lambda| \geq S$. For $|\lambda| \leq S$ we use the fact that $J_{N}=\{1\}$, and so $R_{0}, \ldots, R_{N}$ have no common zeroes. So we just put $\beta=\min _{|\lambda| \leq S} M(\lambda)>$ 0 . We conclude that for any $\lambda$ we have

$$
M(\lambda) \geq \gamma\left(1+|\lambda|^{d}\right),
$$

where $\gamma=\frac{1}{2} \min \left\{2 K, \beta, \frac{1}{S}\right\}$. Combining (4.2) and (4.3) we obtain for each $k \geq N+1$ and for each $\lambda$ the bound $\left|R_{k}(\lambda)\right| \leq C S(k) M(\lambda)$, with $C=\frac{1}{\gamma}$. This completes the proof of Theorem 4.2.

4.2. An example: polynomial moments. Consider the Stieltjes transform of a given polynomial $q(x)=\sum_{i=0}^{d} \alpha_{i} x^{i}$ :

$$
S_{\lambda}(q)(z)=\int_{0}^{\lambda} \frac{q(x) d x}{1-x z}=\sum_{k=0}^{\infty} m_{k}(\lambda, q) z^{k}, \quad \lambda \in \mathbb{C},
$$

where $m_{k}(\lambda, q)=\int_{0}^{\lambda} x^{k} q(x) d x$ are the moments of $q$ on $[a, b]$. Explicit computations show that $S_{\lambda}(q)(z)=G(x, \lambda)-\frac{q(z)}{z} \log (1-\lambda z)$, with $G(x, \lambda)$ a polynomial in $\lambda$ and $z$. However, obtaining uniform Taylor domination for $S_{\lambda}(q)$ is not completely straightforward. Indeed, as we shall see in Section 5 below, already for rational functions uniform Taylor domination is a nontrivial fact, which is, essentially, equivalent to the classical Turán Lemma in Harmonic Analysis $([\mathbf{2 3}, \mathbf{2 4}])$. Analysing the explicit formula for $S_{\lambda}(q)(z)$ given above we encounter roughly the 
same difficulties. So we shall obtain uniform Taylor domination for $S_{\lambda}(q)$ via Theorem 4.2. Without loss of generality we can assume that $q$ has degree exactly $d$, i.e. $\alpha_{d} \neq 0$. To simplify a presentation we also shall assume that $q(0) \neq 0$, i.e. $\alpha_{0} \neq 0$.

Proposition 4.1. The Stieltjes transform $S_{\lambda}(q)(z)$ satisfies conditions (Afam), and possesses $\left(\frac{1}{\lambda}, d, C\right)$-uniform Taylor domination.

Proof: For $q$ as above we immediately get $m_{k}(\lambda, q)=\lambda^{k+1} q_{k}(\lambda)$, where $q_{k}(\lambda)=\sum_{i=0}^{d} \alpha_{i, k} \lambda^{i}$ with $\alpha_{i, k}=\frac{1}{k+i} \alpha_{i}$. We put $P(\lambda)=\lambda$, and $R_{k}(\lambda)=$ $q_{k}(\lambda)$. Clearly, $q_{k}(\lambda)$ are polynomials of degree $d$, and their coefficients decrease in $k$. So we can put $S(k) \equiv C_{0}$, for $C_{0}=\|q\|$. The stabilization moment of the ideals $J_{k}=\left\{q_{0}, \ldots, q_{k}\right\}$ can also be easily estimated. Indeed, the moments $m_{k}(\lambda)$ are the scalar products $m_{k}=\left\langle x^{k}, q(x)\right\rangle$ on the interval $[0, \lambda]$. Since the system $1, x, \ldots, x^{d}$ in the space $\mathcal{P}_{d}$ of polynomials of degree $d$ on $[0, \lambda]$ is linearly independent for each $\lambda \neq 0$, the moments $m_{k}(\lambda)$ cannot vanish simultaneously for any such $\lambda$. Therefore the polynomials $q_{0}, \ldots, q_{d}$ have no common zeroes (at $\lambda=0$ they do not vanish by assumptions). Hence $J_{d}=\{1\}$, and $N \leq d$.

It remains to show that for each $\lambda \in \mathbb{C}$ we have $\lim _{\sup _{k \rightarrow \infty}}\left|q_{k}(\lambda)\right|^{\frac{1}{k}}=$ 1. This is equivalent to the equality $R(\lambda)=|\lambda|^{-1}$ for the radius of convergence of the series in (4.4). But this fact follows directly from the integral formula for the Stieltjes transform in (4.4): the nearest to zero singularity of $S_{\lambda}(q)(z)$ is at $z=\frac{1}{\lambda}$. Thus conditions (Afam-1) and (Afam-2) are satisfied. By the remark above, in the case of scalar $\lambda$ conditions (Afam-1) and (Afam-2), imply (Afam-3). This completes the proof.

Remark. Computations of the scalar products above can be performed in a more "effective" way, showing, in particular, that the constant $C$ in Taylor domination for $S_{\lambda}(q)(z)$ depends only on the degree of $q(x)$. Compare the results on uniform Taylor domination for Stieltjes transforms of $D$-finite functions in [1]. Also the condition $\limsup _{k \rightarrow \infty}\left|q_{k}(\lambda)\right|^{\frac{1}{k}}=1$ can be verified directly: the polynomials $q_{k}(\lambda)=\sum_{i=0}^{d} \alpha_{i, k} \lambda^{i}$ with $\alpha_{i, k}=\frac{1}{k+i} \alpha_{i}$ can be considered for $k \rightarrow \infty$ as perturbations of $\frac{1}{k} q(\lambda)$, and their behavior near the roots of $q$ can be described rather accurately.

Theorem 4.2 can be applied also to one specific case of the Poincaré map for the Abel equation on the interval $[0, \lambda]$, namely, to the equation $y^{\prime}=y^{2}+c x y^{3}$ considered by Liouville [19]. This equation can be integrated explicitly, but the study of its Poincaré map is still far from being straightforward (see $[\mathbf{8}, \mathbf{1 3}])$. We believe that application of the Bautin 
approach in this case may be instructive, and plan to present some results in this direction separately. However, it is important to stress that in general conditions (Afam) are not satisfied for the Poincaré map of Abel equations.

\section{Turán Lemma and its extensions}

The most basic example of uniform Taylor domination concerns Taylor coefficients of rational functions. It is provided by the classical Turán Lemma (see $[\mathbf{2 3}, \mathbf{2 4}]$ ). Consider a linear recurrence relation with constant coefficients

$$
a_{k}=\sum_{j=1}^{d} c_{j} a_{k-j}, \quad k=d, d+1, \ldots .
$$

Given $\bar{a}=\left(a_{0}, \ldots, a_{d-1}\right)$ the entire sequence $a_{k}, k=0,1, \ldots$, is uniquely determined by (5.1). For each $\bar{a}=\left(a_{0}, \ldots, a_{d-1}\right)$ the generating function $f(z)=f_{\bar{a}}(z)=\sum_{k=0}^{\infty} a_{k} z^{k}$ of the recurrence (5.1) is a rational function of the form $f(z)=\frac{P(z)}{Q(z)}$, with $Q(z)=1-\sum_{j=1}^{d} c_{j} z^{j}$ being the characteristic polynomial of $(5.1)$, and with $\operatorname{deg} P(z) \leq d-1$. Conversely, for each rational function $f(z)=\frac{P(z)}{Q(z)}=\sum_{k=0}^{\infty} a_{k} z^{k}$, with $Q$ as above and $\operatorname{deg} P(z) \leq d-1$, its Taylor coefficients $a_{k}$ satisfy (5.1). We consider $f(z)$ as an A-family, the parameters $\lambda$ being the coefficients $c_{j}$ of (5.1), and the initial values $\bar{a}=\left(a_{0}, \ldots, a_{d-1}\right)$, or, alternatively, the coefficients $c_{j}$ and the coefficients of $P(z)$. We shall omit these parameters $\lambda$ in the notations below.

Let $z_{1}, \ldots, z_{d}$ be all the roots of $Q(z)$. By the form of $Q$ we have $z_{i} \neq 0$, $i=1, \ldots, d$, so put $x_{i}=z_{i}^{-1}$. Assume also (to simplify the expressions) that $z_{i} \neq z_{j}$ for $i \neq j$. We can represent our rational function $f(z)$ as a sum of elementary fractions $R(z)=\sum_{i=1}^{d} \frac{\alpha_{i}}{1-x_{i} z}$, so expanding geometric progressions we get the Taylor coefficients $a_{k}$ of $f(z)$ as the values of the exponential polynomial $\psi(s)=\sum_{i=1}^{d} \alpha_{i} x_{i}^{s}$ at the integer points $s=k$.

Put $\rho=\max _{i=1, \ldots, n}\left|x_{i}\right|$ and let $R=\frac{1}{\rho}=\min _{i=1, \ldots, n}\left|z_{i}\right|$ be the radius of convergence of $f(z)=\frac{P(z)}{Q(z)}=\sum_{k=0}^{\infty} a_{k} z^{k}$.

Theorem 5.1 (Turán, $[23,24]$ ). For each $k \geq d+1$

$$
a_{k} R^{k} \leq\left[\frac{4 e(k+d-1)}{d}\right]^{d-1} \max _{i=1, \ldots, d}\left|a_{i}\right| R^{i} .
$$

This theorem provides a uniform Taylor domination for rational functions in their maximal disk of convergence $D_{R}$. Indeed, after rescaling 
to $D_{1}$ the parameters of $(5.2)$ depend only on the degree of the rational function $f(z)$, but not on its specific coefficients. We present below, for more general "Poincaré-type" linear recurrence relations, a weaker version of Turán Lemma, proved in $[\mathbf{1}]$. It provides uniform in parameters Taylor domination not in the maximal disk of convergence, but in its concentric sub-disk of a sufficiently small radius.

5.1. Poincaré-type recurrence relations. We consider linear recur-

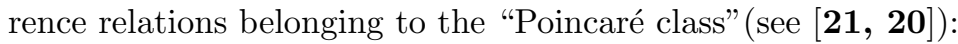

$$
a_{k}=\sum_{j=1}^{d}\left[c_{j}+\psi_{j}(k)\right] a_{k-j}, \quad k=d, d+1, \ldots, \quad \lim _{k \rightarrow \infty} \psi_{j}(k)=0 .
$$

It is well known that Taylor coefficients of solutions of linear ODE's with polynomial coefficients obey recurrence relations of the form (5.3), with $\psi_{j}(k)$ rational functions in $k$. The same is true for the series arising at regular singular points of such ODE's. However, below we shall not use this special information, and we shall allow general sequences $\psi_{j}(k)$, tending to zero as $k$ tends to infinity.

Let $Q(z)=1-\sum_{j=1}^{d} c_{j} x^{j}$ be the characteristic polynomial of (5.3), and let $z_{1}, \ldots, z_{d}$ be all the roots of $Q(z)$. As above, we put $x_{j}=z_{j}^{-1}$, denote by $\rho$ the maximal absolute value of $x_{j}$, and put $R=\frac{1}{\rho}$.

Let us now define $\widehat{N}$ as the first index such that for $k \geq \widehat{N}+1$ we have $\left|\psi_{j}(k)\right| \leq 2^{d} \rho^{j}$, and put $N=\widehat{N}+d$.

Theorem $5.2([\mathbf{1}])$. Let $a_{0}, a_{1}, \ldots$ satisfy (5.3). Then for each $k \geq N+1$ we have

$$
\left|a_{k}\right| R^{k} \leq 2^{(d+3) k} \max _{j=0, \ldots, N}\left|a_{j}\right| R^{j} .
$$

Theorem 5.2 provides Taylor domination in a disk of radius $R^{\prime}=\frac{R}{2^{d+3}}$, and it is uniform in the geometry of the roots $z_{1}, \ldots, z_{d}$. As far as the dependence of $N$ on the behavior of $\psi_{j}(k)$ is concerned, it is essentially sharp. It would be desirable to extend this Taylor domination to the full disk of convergence. Some results in this direction are obtained in $[\mathbf{1}]$, based on the Poincaré-Perron Theorem (see $[\mathbf{2 1}, \mathbf{2 0}]$ ), and on its recent extensions. However, the dependence of the constants on the geometry of the roots $z_{1}, \ldots, z_{d}$ and on $\psi_{j}(k)$ becomes less explicit. We believe that more can be done in this direction.

\section{References}

[1] D. Batenkov and Y. Yomdin, Taylor domination, Turán lemma, and Poincaré-Perron sequences, submitted. 
[2] N. BAutin, Du nombre de cycles limites naissant en cas de variation des coefficients d'un état d'équilibre du type foyer ou centre, $C$. $R$. (Doklady) Acad. Sci. URSS (N. S.) 24 (1939), 669-672.

[3] N. N. Bautin, On the number of limit cycles which appear with the variation of coefficients from an equilibrium position of focus or center type, American Math. Soc. Translation 100 (1954), 19 pp.

[4] M. BiERnACKI, Sur les fonctions multivalentes d'ordre $p$, C. R. Acad. Sci. (Paris) 203 (1936), 449-451.

[5] L. DE Branges, A proof of the Bieberbach conjecture, Acta Math. 154 (1-2) (1985), 137-152. DOI: 10.1007/BF02392821.

[6] M. Briskin, N. Roytvarf, and Y. Yomdin, Center conditions at infinity for Abel differential equations, Ann. of Math. (2) 172(1) (2010), 437-483. DOI: 10.4007/annals. 2010.172.437.

[7] M. BRiskin AND Y. Yomdin, Algebraic families of analytic functions. I, J. Differential Equations 136(2) (1997), 248-267. DOI: 10.1006/jdeq.1996.3250.

[8] J.P. Francoise, N. Roytvarf, and Y. Yomdin, Analytic continuation and fixed points of the Poincaré mapping for a polynomial Abel equation, J. Eur. Math. Soc. (JEMS) 10(2) (2008), 543-570. DOI: $10.4171 /$ JEMS/122.

[9] J.-P. Francoise AND Y. Yomdin, Bernstein inequalities and applications to analytic geometry and differential equations, J. Funct. Anal. 146(1) (1997), 185-205. DOI: 10.1006/jfan.1996.3029.

[10] J.-P. Francoise And Y. Yomdin, Projection of analytic sets and Bernstein inequalities, in: "Singularities Symposium-Eojasiewicz 70" (Kraków, 1996; Warsaw, 1996), Banach Center Publ. 44, Polish Acad. Sci., Warsaw, 1998, pp. 103-108.

[11] A. Gasull, De les equacions diferencials d'Abel al problema XVI de Hilbert, Butl. Soc. Catalana Mat. 28(2) (2013), 123-146. DOI: 10.2436/20.2002.01.49.

[12] A. Gasull And J. Llibre, Limit cycles for a class of Abel equations, SIAM J. Math. Anal. 21(5) (1990), 1235-1244. DOI: 10.1137/ 0521068.

[13] Sh. Giat, Y. Shelah, C. Shikelman, and Y. Yomdin, Algebraic geometry of Abel differential equation, Rev. R. Acad. Cienc. Exactas Fís. Nat. Ser. A Math. RACSAM 108(1) (2014), 193-210. DOI : $10.1007 / \mathrm{s} 13398-012-0112-4$.

[14] J. Giné, M. Grau, And J. Llibre, Universal centres and composition conditions, Proc. London Math. Soc. (3) 106(3) (2013), 481-507. DOI : 10.1112/plms/pds050. 
[15] A. W. Goodman, On some determinants related to $p$-valent functions, Trans. Amer. Math. Soc. 63 (1948), 175-192.

[16] A. W. Goodman, Open problems on univalent and multivalent functions, Bull. Amer. Math. Soc. 74 (1968), 1035-1050.

[17] W. K. Hayman, "Multivalent functions", Second edition, Cambridge Tracts in Mathematics 110, Cambridge University Press, Cambridge, 1994. DOI : 10.1017/CB09780511526268.

[18] A. Lins Neto, On the number of solutions of the equation $\frac{d x}{d t}=$ $\sum_{j=0}^{n} a_{j}(t) x^{j}, 0 \leq t \leq 1$, for which $x(0)=x(1)$, Invent. Math. 59(1) (1980), 67-76. DOI : 10.1007/BF01390315.

[19] R. Liouville, Sur une équation différentielle du premier ordre, Acta Math. 27(1) (1903), 55-78. DOI: 10.1007/BF02421296.

[20] O. Perron, Über Summengleichungen und Poincarésche Differenzengleichungen, Math. Ann. 84(1-2) (1921), 1-15. DOI: 10.1007/BF01458689.

[21] H. Poincaré, Sur les equations linéaires aux différentielles ordinaires et aux différences finies, Amer. J. Math. 7(3) (1885), 203-258. DOI : $10.2307 / 2369270$.

[22] N. Roytwarf and Y. Yomdin, Bernstein classes, Ann. Inst. Fourier (Grenoble) 47(3) (1997), 825-858.

[23] P. Turán, "Über eine neue Methode der Analysis und deren Anwendungen", Akadémiai Kiadó, Budapest, 1953.

[24] P. TuRÁN, "On a new method of analysis and its applications", With the assistance of G. Halász and J. Pintz, With a foreword by Vera T. Sós, Pure and Applied Mathematics (New York), A WileyInterscience Publication, John Wiley \& Sons, Inc., New York, 1984.

[25] Y. Yomdin, Global finiteness properties of analytic families and algebra of their Taylor coefficients, in: "The Arnoldfest" (Toronto, ON, 1997), Fields Inst. Commun. 24, Amer. Math. Soc., Providence, RI, 1999, pp. 527-555.

Department of Mathematics

The Weizmann Institute of Science

Rehovot 76100

Israel

E-mail address: yosef.yomdin@weizmann.ac.il 